University of Nebraska - Lincoln

DigitalCommons@University of Nebraska - Lincoln

\title{
Outcrossing and crossbreeding recovers deteriorated traits in laboratory cultured Steinernema carpocapsae nematodes
}

John M. Chaston

Brigham Young University

Adler R. Dillman

Brigham Young University

David I. Shapiro-llan

USDA-ARS

Anwar L. Bilgrami

Rutgers University

Randy Gaugler

Rutgers University

See next page for additional authors

Follow this and additional works at: https://digitalcommons.unl.edu/usdaarsfacpub

Part of the Agricultural Science Commons

Chaston, John M.; Dillman, Adler R.; Shapiro-Ilan, David I.; Bilgrami, Anwar L.; Gaugler, Randy; Hopper, Keith R.; and Adams, Byron J., "Outcrossing and crossbreeding recovers deteriorated traits in laboratory cultured Steinernema carpocapsae nematodes" (2011). Publications from USDA-ARS / UNL Faculty. 842. https://digitalcommons.unl.edu/usdaarsfacpub/842

This Article is brought to you for free and open access by the U.S. Department of Agriculture: Agricultural Research Service, Lincoln, Nebraska at DigitalCommons@University of Nebraska - Lincoln. It has been accepted for inclusion in Publications from USDA-ARS / UNL Faculty by an authorized administrator of DigitalCommons@University of Nebraska - Lincoln. 


\section{Authors}

John M. Chaston, Adler R. Dillman, David I. Shapiro-llan, Anwar L. Bilgrami, Randy Gaugler, Keith R. Hopper, and Byron J. Adams 


\title{
Outcrossing and crossbreeding recovers deteriorated traits in laboratory cultured Steinernema carpocapsae nematodes
}

\author{
John M. Chaston a,1,2, Adler R. Dillman a,1,3, David I. Shapiro-Ilan ${ }^{\mathrm{b}}$, Anwar L. Bilgrami ${ }^{\mathrm{c}}$, Randy Gaugler ${ }^{\mathrm{c}}$, \\ Keith R. Hopper ${ }^{\mathrm{d}}$, Byron J. Adams ${ }^{\mathrm{e}, *}$ \\ ${ }^{a}$ Microbiology E Molecular Biology Department, and Evolutionary Ecology Laboratories, Brigham Young University, Provo, UT 84602-5253, USA \\ ${ }^{\mathrm{b}}$ SE Fruit and Tree Nut Research Unit, USDA-ARS, SAA, Byron, GA 31008, USA \\ ${ }^{c}$ Center for Vector Biology, Rutgers University, New Brunswick, NJ 08901, USA \\ d Department of Entomology and Wildlife Ecology, University of Delaware, Newark, DE 19716, USA \\ e Department of Biology, and Evolutionary Ecology Laboratories, Brigham Young University, Provo, UT 84602, USA
}

\section{A R T I C L E I N F O}

Article history:

Received 29 October 2010

Received in revised form 17 February 2011

Accepted 18 February 2011

Available online 9 April 2011

\section{Keywords:}

Biological control

Entomopathogenic nematodes

Inbreeding depression

Steinernema carpocapsae

Trait deterioration

\begin{abstract}
A B S T R A C T
The nematode Steinernema carpocapsae infects and kills many pest insects in agro-ecosystems and is commonly used in biocontrol of these pests. Growth of the nematodes prior to distribution for biocontrol commonly results in deterioration of traits that are essential for nematode persistence in field applications. To better understand the mechanisms underlying trait deterioration of the efficacy of natural parasitism in entomopathogenic nematodes, we explored the maintenance of fitness related traits including reproductive capacity, heat tolerance, virulence to insects and 'tail standing' (formerly called nictation) among laboratory-cultured lines derived from natural, randomly mating populations of $S$. carpocapsae. Laboratory cultured nematode lines with fitness-related trait values below wild-type levels regained wild-type levels of reproductive and heat tolerance traits when outcrossed with a non-deteriorated line, while virulence and 'tail standing' did not deteriorate in our experiments. Crossbreeding two trait-deteriorated lines with each other also resulted in restoration of trait means to wild-type levels in most crossbred lines. Our results implicate inbreeding depression as the primary cause of trait deterioration in the laboratory cultured S. carpocapsae. We further suggest the possibility of creating inbred lines purged of deleterious alleles as founders in commercial nematode growth.
\end{abstract}

(ㄷ) 2011 Australian Society for Parasitology Inc. Published by Elsevier Ltd. All rights reserved.

\section{Introduction}

Parasitism has arisen independently at least seven times in the phylum Nematoda, with animal parasitism having arisen at least four times (Blaxter et al., 1998). Nematodes that have evolved to engage symbiotic enterobacteria in insect endoparasitism are called entomopathogenic nematodes (EPNs) and this type of parasitism has arisen at least twice within the phylum Nematoda (Adams et al., 2006). EPNs kill infected insect hosts within 24-48 h p.i. (Poinar, 1990), making them beneficial for use in biological control (the practice of using natural enemies to control endemic or exotic pests). Steinernema carpocapsae is a model EPN due to its cosmopolitan distribution, broad host range and high

\footnotetext{
* Corresponding author. Tel.: +1 801422 3132; fax: +1 8014220090.

E-mail address: bjadams@byu.edu (B.J. Adams).

1 These authors contributed equally to this work.

2 Present address: Department of Bacteriology, University of Wisconsin-Madison, Madison, WI 53706, USA.

3 Present address: Division of Biology, California Institute of Technology, Pasadena, CA 91125, USA.
}

tolerance of environmental extremes (desiccation, hypoxia, UV, heat and cold tolerance (Grewal, 2002; Hominick, 2002)). Steinernema carpocapsae nematodes associate with and carry Xenorhabdus nematophila bacteria in a specialized structure called the receptacle, located at the anterior end of the nematode intestine of the infective juvenile nematode life stage (Bird and Akhurst, 1983; Martens et al., 2003; Snyder et al., 2007). Xenorhabdus nematophila bacteria provide nutrition to the nematode and assist the nematode in killing infected insects. The nematode penetrates into the haemocoel of a potential insect host (Poinar, 1990) where it releases the bacteria. S. carpocapsae nematodes are gonochoristic (reproducing via males and females) and can only reproduce when both sexes infect the same host. After two to three generations of reproduction, depending on the size of the host and the founding population, unknown cues (possibly high nematode density and nutrient depletion, Popiel et al., 1989) cause most of the nematodes to develop into the infective juvenile life stage. Infective juvenile progeny are non-feeding, developmentally arrested L3s, and are encased in an environmentally protective cuticle. Infective juveniles emerge from a resource-depleted cadaver in search of a new insect host. 
Entomopathogenic nematodes are commonly employed against insect pests in agroecosytems (Gaugler and Kaya, 1990; Kaya et al., 2006). In field applications entomopathogenic nematodes induce target insect mortality with $0-100 \%$ efficacy across a wide variety of environments (Shapiro-Ilan et al., 2002). Varying rates of insect mortality likely result from a variety of factors, including compatibility of the EPN and insect host, environmental conditions, and the timing of application. Another factor that reduces the ability of EPNs to kill their insect hosts is trait deterioration in parasitism and other fitness-related traits that occurs during repeated culturing in laboratory or industrial settings. Trait deterioration after laboratory rearing has been reported for heat tolerance (Shapiro et al., 1996; Bilgrami et al., 2006), longevity (Gaugler and Campbell, 1991), infectivity, sex ratio, reproductive capacity (Stuart and Gaugler, 1996; Bilgrami et al., 2006) and virulence and 'tail standing' (Bai et al., 2005; Bilgrami et al., 2006). The causes of trait deterioration, or practices that can minimize or reduce trait deterioration, are unknown.

Trait change under conditions of mass production may result from genetic processes including inbreeding depression or inadvertent selection (Hopper et al., 1993). In S. carpocapsae, inbreeding depression is a likely cause of trait deterioration during repeated laboratory culture due to its mating style. Gonochoristic organisms rely upon sexual recombination to mask deleterious alleles that arise from mutation at low frequencies in a population and are more sensitive to inbreeding depression than are selfing species (e.g. Dolgin et al., 2007). Other genetic processes may also be at work, such as inadvertent selection of nematode lines for phenotypes that are beneficial under conditions of mass production but deleterious under field conditions. Also, non-genetic factors such as disease may contribute to trait deterioration under mass production conditions.

Our objective was to determine the underlying causes of deterioration in EPNs, hoping to inform nematode production for biocontrol programs. In this study we used six nematode lines (experimental lines 1-5 and a control line) reported previously (Bilgrami et al., 2006). All of the lines were derived from the same parental line by the approach shown in Fig. 1 (some of the lines were developed as part of an earlier study by Bilgrami et al., 2006). Two different $S$. carpocapsae populations were isolated from two different sites (in New Jersey and Arkansas, USA) and mixed together to create the base line. The base line was divided into six groups called experimental lines 1-5 and the control line, which were kept as separate cultures thereafter. The experimental lines were serially passed through Galleria mellonella 20 times as completely separate lines. The control line was passed once per five experimental line passes in order to maintain stocks that were viable but had undergone fewer rounds of reproduction. For each passage the infective juveniles that emerged from an insect infection were used to infect a new G. mellonella pool and the previous generation was discarded. Bilgrami et al. (2006) showed that the experimental lines showed reduced trait values for reproductive capacity, heat tolerance, virulence and 'tail standing' (Bilgrami et al., 2006). Trait values of the control line after five passages were similar to the trait values displayed by the lines prior to laboratory culture (Bilgrami et al., 2006). As noted above, most emergent infective juveniles are F3 progeny (Wang and Bedding, 1996), so a single passage represents approximately three generations.

The traits measured by Bilgrami et al. (2006) were direct and indirect indicators of field efficacy. 'Tail standing' (formerly called nictation but see Kruitbos and Wilson, 2010 for semantic revision), a predatory behavior requisite to jumping, and virulence are direct measures of traits important for field efficacy, and the virulence assay approach used has been shown to have good correlation with field efficacy trials (Shapiro-Ilan et al., 2002; Grewal et al., 2005). Reproductive capacity and heat tolerance are not directly related to insect virulence but are important for commercial distribution programs. Lower in vitro reproduction reduces the production capacity of commercial distributors and lower in vivo reproduction reduces nematode persistence following application. Heat tolerance is also important since temperature is a confounding issue for nematode storage, transport, commercial production and field persistence (Shapiro-Ilan and Gaugler, 2002; Shapiro-Ilan et al., 2002; Grewal et al., 2005; Schmeige, 1963). As always, good correlation with field performance can only be confirmed by field trials.

We hypothesize a model where trait deterioration in these laboratory cultured nematode lines has genetic causes resulting from inbreeding of founding populations. If so, outcrossing laboratory cultured nematodes with nematodes isolated in the wild should lead to recovery or an increase in trait values. If trait deterioration results from genetic changes, deteriorated traits could be recovered in progeny of the trait-deteriorated lines by outbreeding trait-deteriorated lines with an undeteriorated line. Alternatively, if non-genetic factors are responsible for trait deterioration, outbred progeny should have similar fitness trait values to their trait-deteriorated ancestral lines. Crossbreeding of inbred lines should assess the causality of inbreeding versus inadvertent selection since recovery of deteriorated traits in crossbred trait deteriorated lines should only be observed if the lines are genetically distinct; if lines were selected towards the same traits, crossbreeding of inbred lines should not result in trait recovery.

\section{Material and methods}

\subsection{Cultures}

In this study we used six nematode lines (experimental lines 1-5 and control line) reported previously (Bilgrami et al., 2006). All of the lines were derived from the same parental line. For all nematode culture in $G$. mellonella, nematode lines were concentrated to $\sim 1000$ nematodes in $250 \mu$ by centrifugation at $0.4 \mathrm{~g}$, and the concentrate was transferred to a \#2 Whatman ${ }^{\circledR}$ filter paper placed inside a $6 \mathrm{~cm}$ petri dish. Subsequently, $10 \mathrm{G}$. mellonella larvae were added to the filter paper, and after 7-10 days the larvae were transferred to a White trap (White, 1927) for collection of infective juveniles. Emergent infective juveniles were stored in water at $15^{\circ} \mathrm{C}$ and one to three nematodes per ml. We passed lines through G. mellonella twice, relative to the output pools of the study by Bilgrami et al. (2006) (Fig. 1), prior to initiating the study in order to obtain healthy working stocks.

Bacterial cultures were isolated directly from insect cadavers. Two days post-nematode infection insects were flame sterilized in ethanol and broken open onto a Luria-Bertani (Difco) or lipid agar (Vivas and Goodrich-Blair, 2001) plate. The plates were incubated overnight at $30^{\circ} \mathrm{C}$ and $\mathrm{X}$. nematophila was isolated by isolation streak or dilution plating. We verified that the cultures were $X$. nematophila by 16 S rDNA sequencing (Adams et al., 2006).

\subsection{Crosses}

Table 1 shows the nematode lines used to create outcross, crossbred and sib-cross lines. Nematode lines were crossed by placing a single unfertilized female nematode with three male nematodes (three males were added to ensure each female was fertilized) on an agar surface seeded with bacteria from the same nematode line as the female (i.e. 1 experimental line 1 female +3 males (control line) + experimental line 1 bacteria). Males and unfertilized females were isolated from $G$. mellonella cadavers infected with the respective nematode line by opening the cadaver into Luria-Bertani broth after 2-6 days, examining the nematodes under $40 \times$ magnification, and isolating and transferring them to 


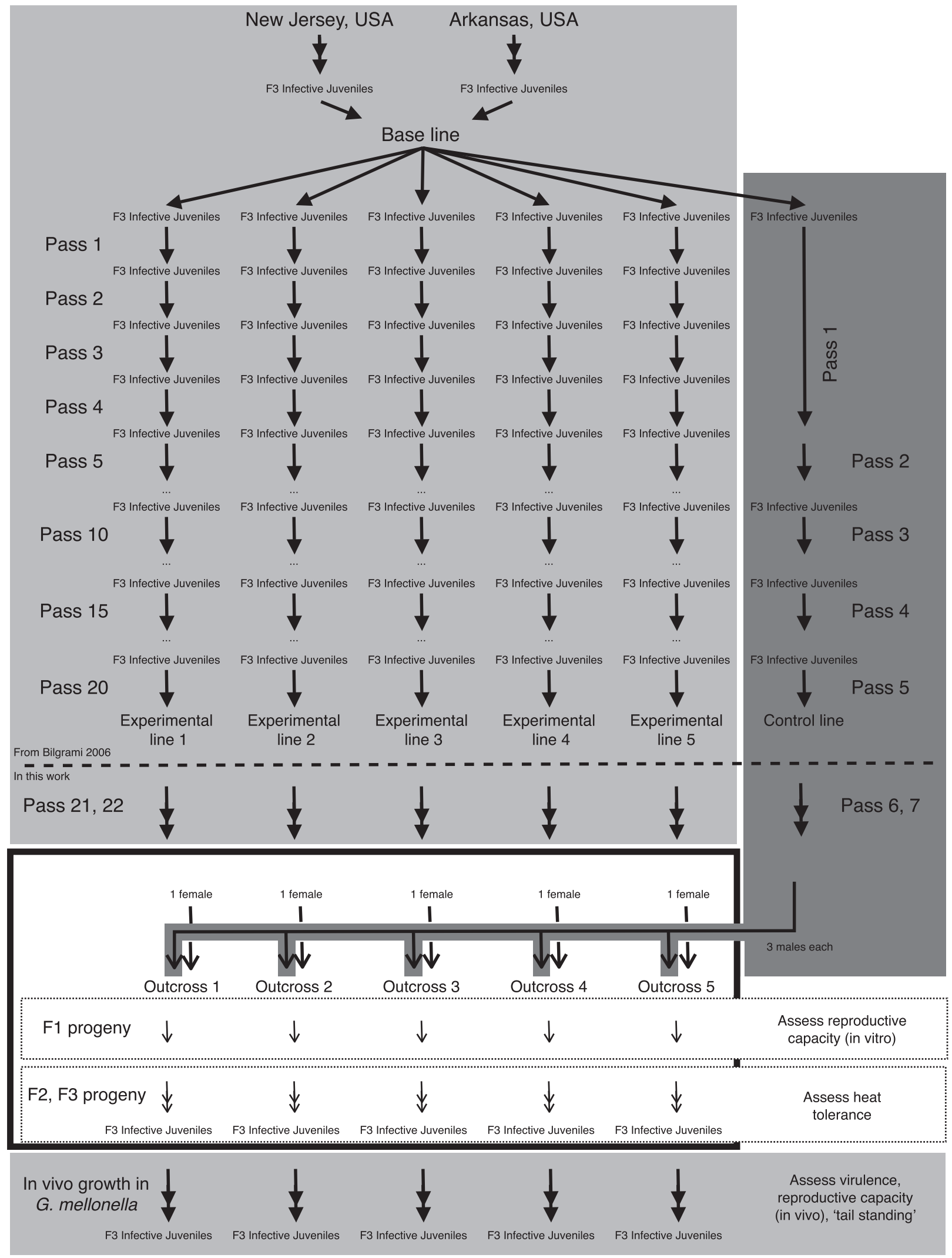

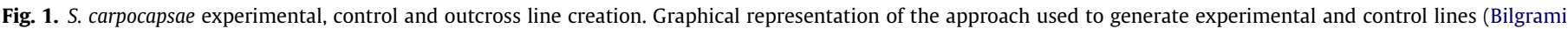

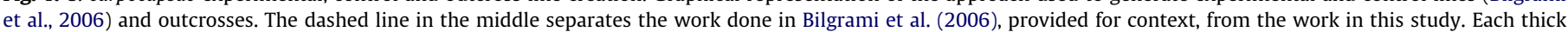

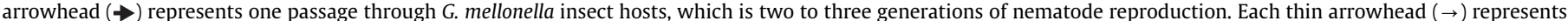

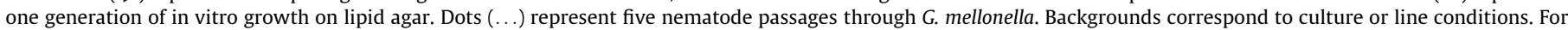

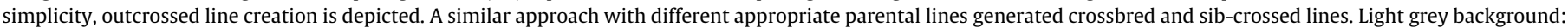

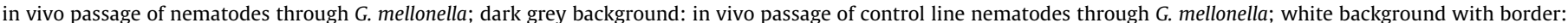
in vitro crossing and reproduction of nematodes on lipid agar. 
Table 1

Design of outcrossed, crossbred and sib-crossed S. carpocapsae lines.

\begin{tabular}{|c|c|c|c|c|}
\hline Cross name & Paternal nematode progenitor & Maternal nematode progenitor & Bacterial symbiont & Number of crossed lines obtained $(\mathrm{N})$ \\
\hline Outcross 1 & Control line & Experimental line 1 & Experimental line 1 & 9 \\
\hline Outcross 2 & Control line & Experimental line 2 & Experimental line 2 & 9 \\
\hline Outcross 3 & Control line & Experimental line 3 & Experimental line 3 & 7 \\
\hline Outcross 4 & Control line & Experimental line 4 & Experimental line 4 & 8 \\
\hline Outcross 5 & Control line & Experimental line 5 & Experimental line 5 & 10 \\
\hline Crossbred A & Experimental line 4 & Experimental line 5 & Experimental line 5 & 9 \\
\hline Crossbred B & Experimental line 5 & Experimental line 4 & Experimental line 4 & 11 \\
\hline Sib-cross 1 & Experimental line 1 & Experimental line 1 & Experimental line 1 & 10 \\
\hline Sib-cross 2 & Experimental line 2 & Experimental line 2 & Experimental line 2 & 8 \\
\hline Sib-cross 3 & Experimental line 3 & Experimental line 3 & Experimental line 3 & 18 \\
\hline Sib-cross 4 & Experimental line 4 & Experimental line 4 & Experimental line 4 & 9 \\
\hline Sib-cross 5 & Experimental line 5 & Experimental line 5 & Experimental line 5 & 8 \\
\hline Sib-cross WT & Control line & Control line & Control line & 12 \\
\hline
\end{tabular}

WT, wild type.

plates with pre-grown bacterial lawns of the appropriate line, which were then incubated at $25^{\circ} \mathrm{C}$. To ensure that the females we transferred were not already fertilized we transferred one late L4 or young adult female nematode to a separate plate for each female nematode that we mixed with male nematodes; none of the lone females produced progeny. On plates where males and females were mixed, development of nematode progeny was assessed visually each day, and a cross was deemed successful if progeny were macroscopically visible within 10 days (progeny should be visible after 5 days). Three types of crosses, outcrosses, crossbreeding crosses and sib-crosses, were performed, and are depicted in tabular (Table 1) and graphical (Fig. 1, outcrosses are representatively shown) forms. Five outcrosses (outcrosses 1-5) were performed by mixing control line males with an experimental line female on agar seeded with experimental line bacteria. Two crossbreeding crosses (crossbred lines A and B) were performed by mixing experimental line 4 males with an experimental line 5 female on experimental line 5 bacteria (crossbred line A), and experimental line 5 males with an experimental line 4 female on experimental line 4 bacteria (crossbred line B). Six sib-crosses (one for each of experimental lines 1-5 and control line, called sib-cross 1-5 and sib-cross wild type (WT), respectively) were performed by placing males and a female from the same nematode line on bacteria isolated from the same nematode line (e.g. experimental line 1 males and female on experimental line 1 bacteria). We explicitly note that these are not self-fertilized crosses, but crosses between two nematodes isolated from the same insect cadaver, which may or may not actually be siblings. We use the term sib-cross for simplicity and to explicitly distinguish our approach from names that might suggest self-fertilization. Also, given the extended laboratory culture time and population bottlenecks that occur during each passage, close genetic relatedness of the isolated nematodes is extremely likely and the term not likely to be misleading. Ten attempted crosses were performed in this manner for each of the lines, except for crossbred line $\mathrm{B}$, which was attempted at least 11 times, the control line ( 15 attempts) and reproductive line 3 (20 attempts). Table 1 only reports the number of successful crosses (crosses from which progeny were obtained).

The in vitro reproductive capacity of the crosses was counted directly on the plate that each cross was performed on. Later generations developed as infective juveniles that were collected in White traps, pooled with other replicate crosses from the same cross type in equal ratios (e.g. all nine outcross 1 replicates) and divided into two portions. One portion was assessed for heat tolerance and the other was passed through $G$. mellonella twice prior to assessing in vivo reproductive capacity, virulence and 'tail standing' (Fig. 1). Outcross, sib-cross and crossbred nematode lines were passed through $G$. mellonella in parallel to control for infective juvenile age (i.e. time post-emergence).

\subsection{Trait assays}

\subsubsection{Reproductive capacity (in vitro)}

Four or 5 days after crossing the nematodes, while the in vitro progeny were in the adult stage of development, we counted the number of nematodes on the surface of the agar plate. These offspring were the F1 progeny of the crosses. All plates yielding crossed progeny ( $n=7-18$ plates) were counted.

\subsubsection{Reproductive capacity (in vivo)}

Reproductive capacity assays were performed as described in Bilgrami et al. (2006). Three cadavers for each line, comprising one replicate, were placed in a White trap. Emerging nematodes were collected for counting 18 days p.i. and represent the F3 progeny of the infecting nematodes and the F12 progeny of the experimental and control line nematodes that were outcrossed, crossbred and sib-crossed in this study. In vivo reproductive capacity was expressed as the number of infective juveniles per insect. Treatments were replicated four times and the entire assay was repeated (two trials). A total of 24 insects were used per treatment.

\subsubsection{Heat tolerance}

Immediately prior to assessing heat tolerance we combined nematodes from replicate crosses at a 1:1 ratio with total 1000 nematodes in $5 \mathrm{ml}$ of tap water. Each mixed line was incubated in a water bath at $37^{\circ} \mathrm{C}$ for $6 \mathrm{~h}$ prior to removal from heat to room temperature $\left(\sim 25^{\circ} \mathrm{C}\right)$. Twenty-four hours later we assessed the mortality of 100 of the assayed nematodes by physical probing and detecting movement of the nematode. Response to the probe or active movement without probing was scored as survival, and lack of observable physical response to the probe was scored as death. The ratio of dead to live nematodes was determined. The assay was conducted twice (two trials) with four replicates per assay.

\subsubsection{Virulence}

Virulence assays were conducted based on procedures described by Bilgrami et al. (2006). Approximately 25 infective juveniles from each line were added to $30 \mathrm{ml}$ cups filled with sand (10\% moisture). Each dish contained four G. mellonella larvae. Non-treated dishes (with water only) served as the control. The dishes were incubated at $25^{\circ} \mathrm{C}$ and insect mortality was assessed after 30 and $48 \mathrm{~h}$. There were five replicate treatments and the entire experiment was repeated (two trials).

\subsection{5. 'Tail standing'}

'Tail standing' in S. carpocapsae was studied using $2 \%$ agar plates ( $9 \mathrm{~cm}$ diameter) in which $0.14 \mathrm{~g}$ of sand ( $<150 \mu \mathrm{m}$ particle size) was scattered over the agar surface to generate a substrate suitable for 'tail standing' (Bilgrami et al., 2006). Fifty nematodes were 
transferred to the plate and the proportion standing on their tails (i.e., elevating $>95 \%$ of their body) was determined after $1 \mathrm{~h}$. Treatments were replicated four times and the entire assay was repeated (two trials).

\subsection{Statistical analyses}

All statistical analyses were run in SAS (SAS Software: Version 8.2. SAS Institute, Cary, NC, USA) at $\alpha=0.05$. A separate ANOVA was used to analyze the effects of line/cross on the means of each trait. If a significant difference was detected, then treatment differences were further elucidated through Fisher's protected least significant differences (LSD) test. For experiments that were repeated in time (all but in vitro reproductive capacity), data from both trials were combined, and variation among trials was accounted for as a block effect. Percentage data ('tail standing', heat tolerance and virulence) were arcsine transformed and numerical data (nematode reproduction) were square-root transformed prior to analyses
(Southwood, 1978); non-transformed means are presented in the figures.

\section{Results}

\subsection{Comparison of trait values in sib-crosses and outcrosses}

A brief summary of specific relationships is presented in the text below.

\subsubsection{Reproductive capacity (in vitro)}

In vitro reproductive capacity differed among sib-crosses and outcrosses $(F=13.16 ; \mathrm{df}=10,97 ; P<0.0001$; Fig. 2$)$. Sib-crosses 1-5 had lower numbers of $\mathrm{F} 1$ progeny compared with sib-cross WT. Outcrosses 3, 4 and 5 produced more F1 progeny than did their sib-cross counterparts (Fisher's LSD). Sib-cross WT produced more progeny than any of the outcrosses.

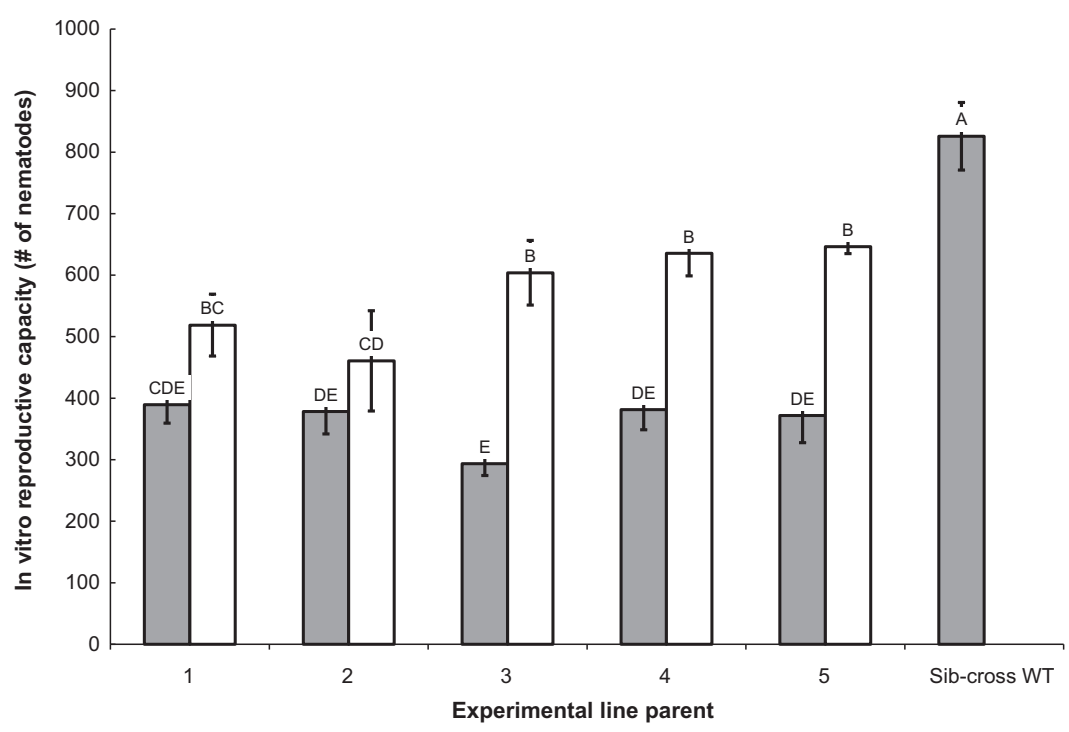

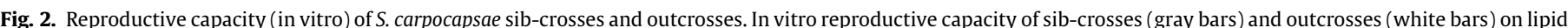

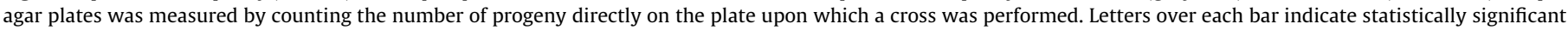
differences between the in vitro reproductive capacity of a given line and all other lines assayed at $\alpha=0.05$. Error bars indicate 1 S.D. from the average of the replicates.

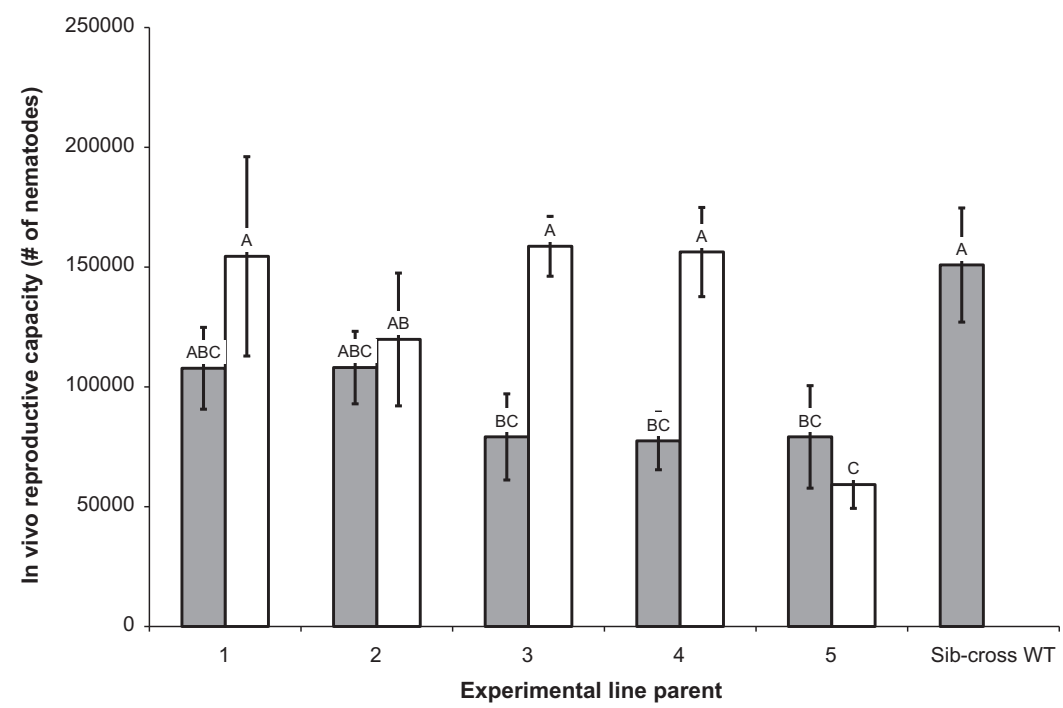

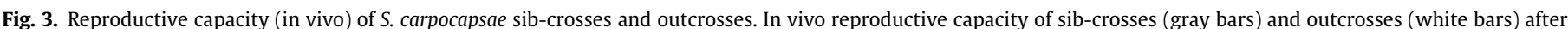

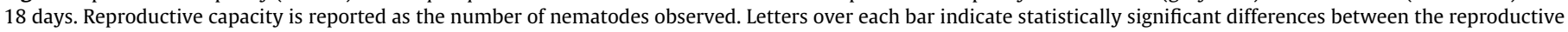
capacity of a given line and all other lines assayed at $\alpha=0.05$. Error bars indicate 1 S.D. from the average of the assayed line. 


\subsubsection{Reproductive capacity (in vivo)}

Sib-crosses 3-5 produced fewer progeny than sib-cross WT, while sib-crosses 1 and 2 produced a similar number to sib-cross WT. Outcrosses 3 and 4 produced more progeny than their counterpart sib-crosses, but in other outcrosses productivity was similar to that of the sib-cross counterpart. Except for outcross 5, outcrosses gave rise to a similar number of progeny as sib-cross WT $(F=3.23 ; \mathrm{df}=10,76 ; P=0.0016$, Fisher's LSD; Fig. 3 ).

\subsubsection{Heat tolerance}

Sib-crosses 2-5 were less resistant to heat than sib-cross WT but sib-cross 1 was similar to sib-cross WT. All the outcrosses were more heat tolerant than their sib-cross counterparts, and as tolerant as sib-cross WT $(F=11.21 ; \mathrm{df}=10,76 ; P<0.0001$, Fisher's LSD; Fig. 4).

\subsubsection{Virulence}

Progeny of all crosses killed all G. mellonella by $48 \mathrm{~h}$, except for sib-cross 1 , which killed $97.5 \%( \pm 2.5 \%, n=10)$ of the larvae to which it was exposed. Even at an earlier time point $(30 \mathrm{~h})$ there were no observable differences between the sib-crosses 1-5 and sib-cross WT $(30 \mathrm{~h}: F=11.75 ; \mathrm{df}=11,48 ; P<0.0001 ; 48 \mathrm{~h}$ : $F=279.1 ; \mathrm{df}=11,107 ; P<0.0001$, Fisher's LSD; Fig. 5). At 30 h outcross 1 killed fewer insects than sib-cross 1 , while outcross 5 killed more larvae than sib-cross 5 . All other outcrosses did not differ in virulence from sib-cross counterparts, and all outcrosses were

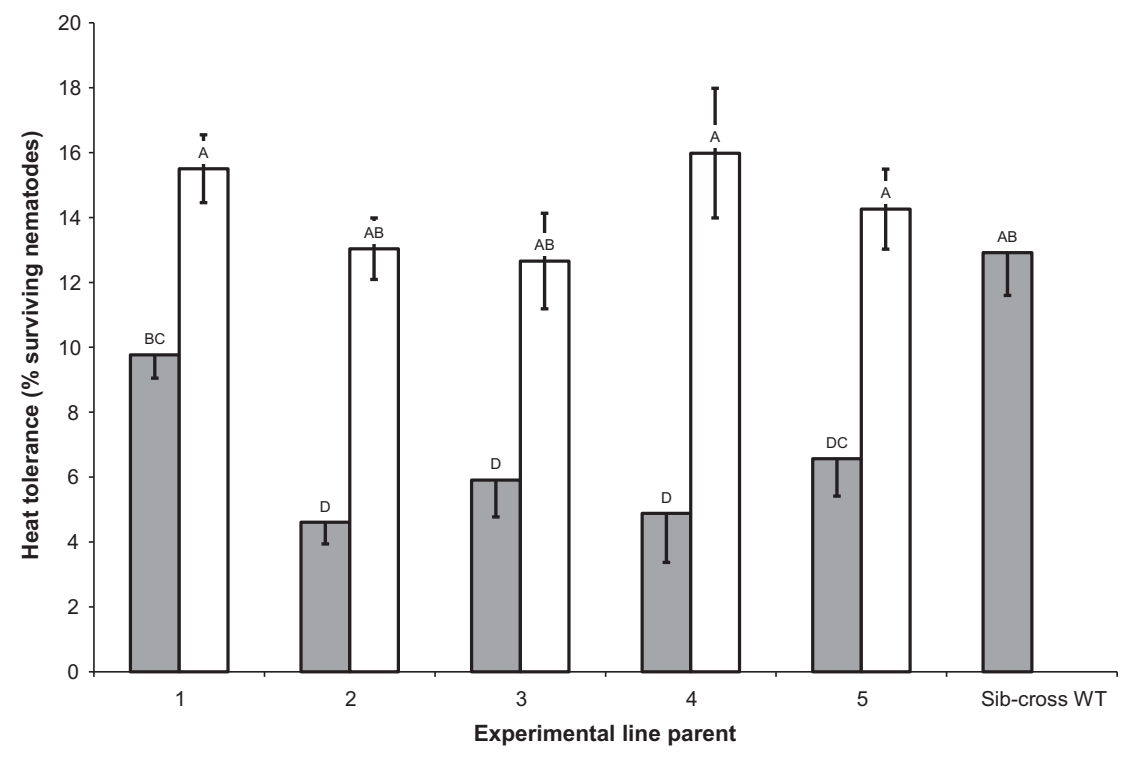

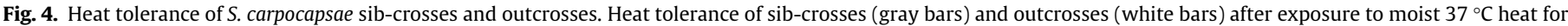

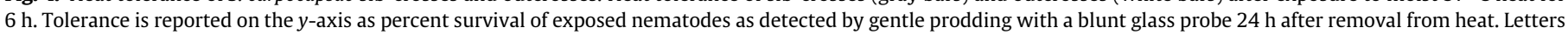

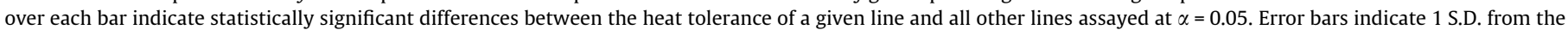
average of the assayed line.

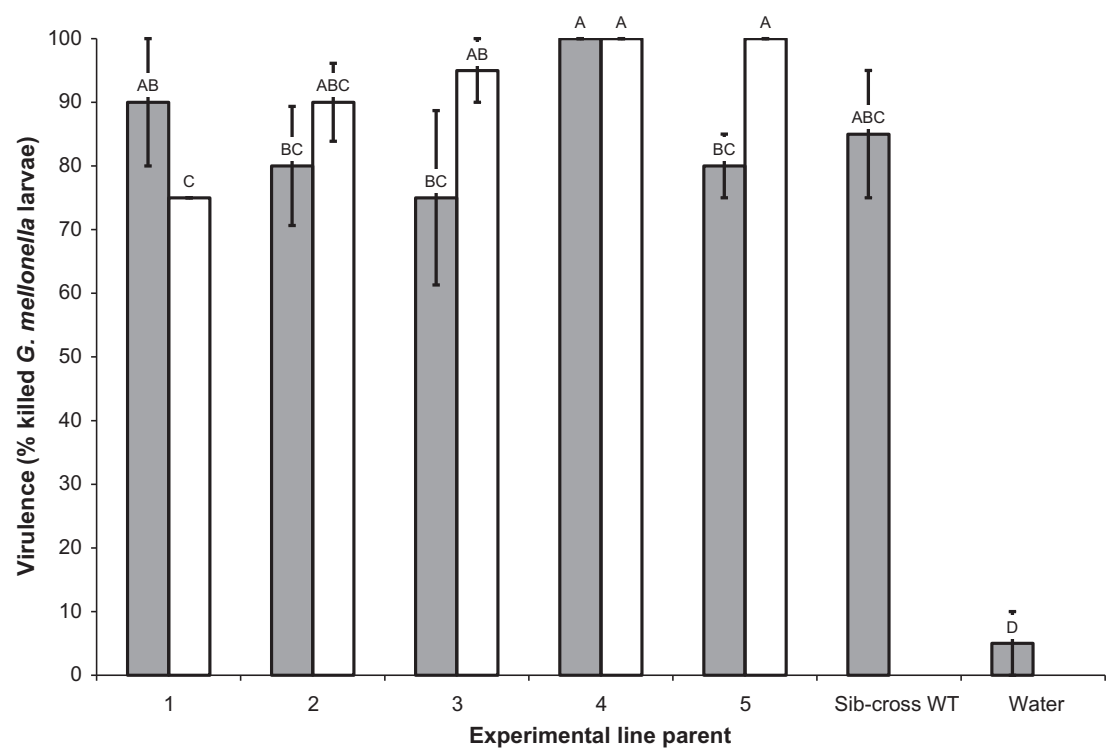

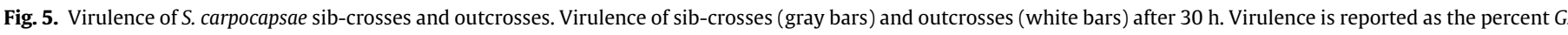

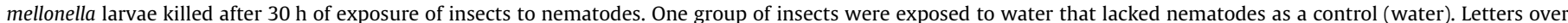

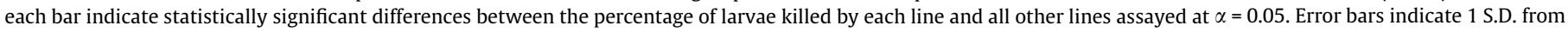
the average of the assayed line. 
similarly virulent to sib-cross WT. Also, all nematode lines produced higher mortality than the control water treatment (which did not exceed 5\% during the experiment).

\subsection{5. 'Tail standing'}

Sib-crosses 1 and 5 displayed 'tail standing' less frequently than sib-cross WT but the other three sib-crosses did not differ from sibcross WT. Only outcross 1 displayed 'tail standing' more frequently than sib-cross 1; all other outcrosses did not differ from the counterpart sib-cross. None of the outcrossed lines differed in 'tail standing' frequency from sib-cross WT $(F=2.17 ; \mathrm{df}=10,76$; $P=0.0285$; Fisher's LSD; Fig. 6).

\subsection{Crossbreeding}

For in vitro and in vivo reproductive capacity $(F=21.13$; $\mathrm{df}=4$, 44; $P<0.0001$; and $F=3.05 ; \mathrm{df}=4,34 ; P=0.030$; Fisher's LSD, respectively) and for heat tolerance (at $30 \mathrm{~h})(F=16.46$; $\mathrm{df}=4$, $34 ; P<0.0001$, Fisher's LSD) crossbred lines A and B both showed increased values with reference to sib-crosses 4 and 5 (Fig. 7). Crossbred line A was less virulent $(F=16.61 ; \mathrm{df}=5,24 ; P<$

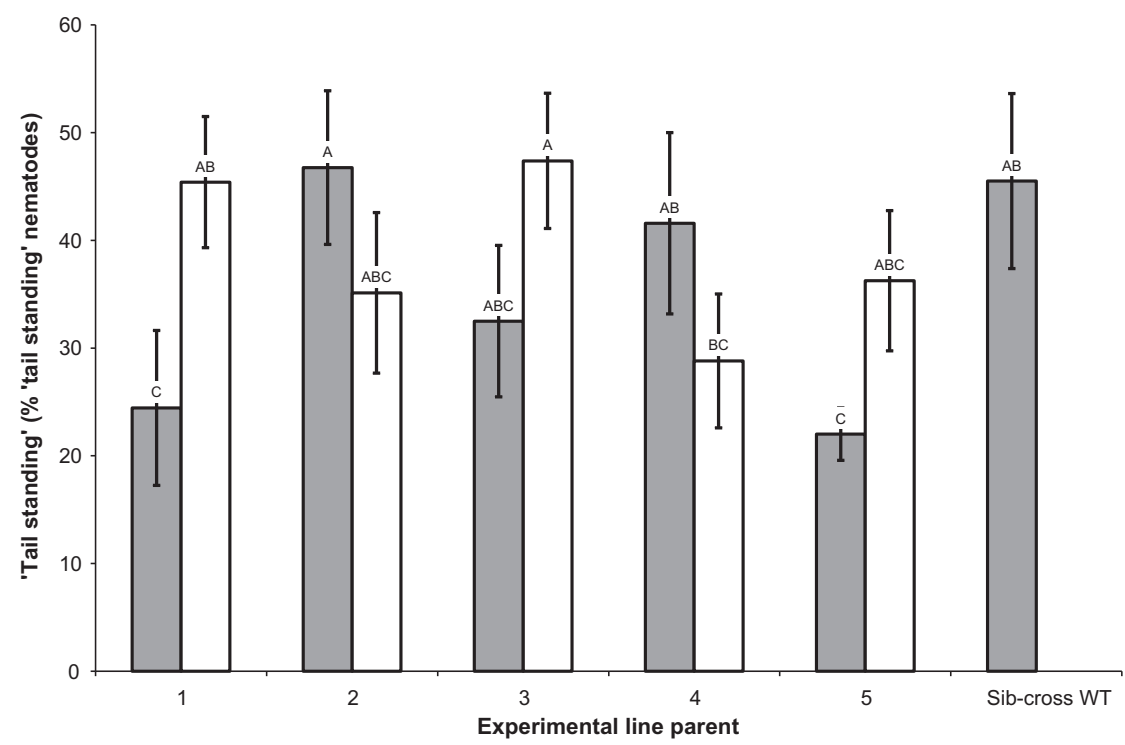

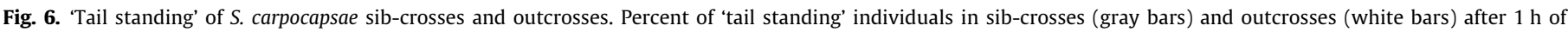

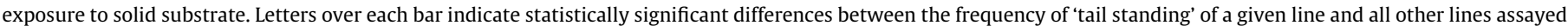
at $\alpha=0.05$. Error bars indicate 1 S.D. from the average of the assayed line.

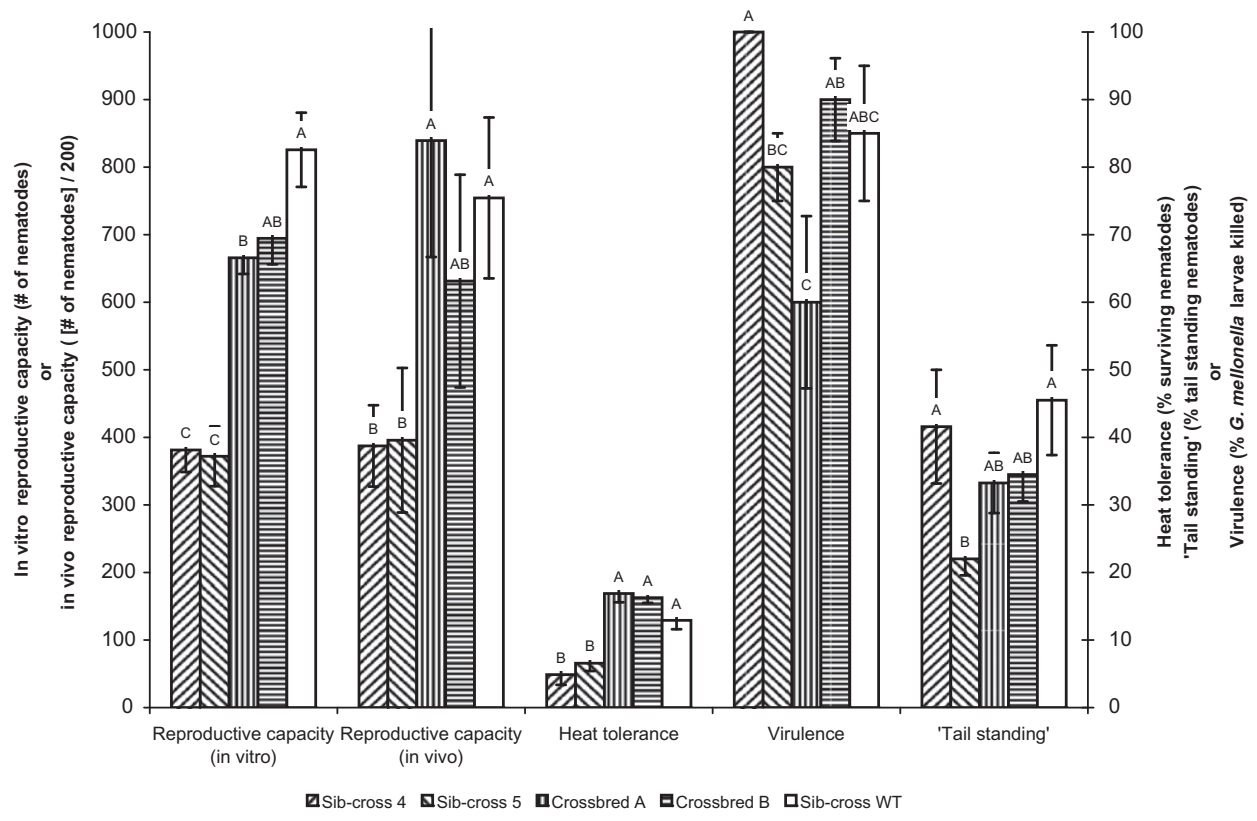

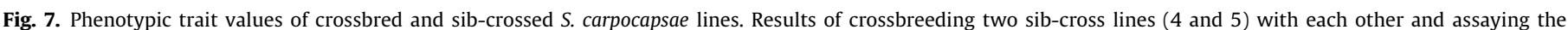

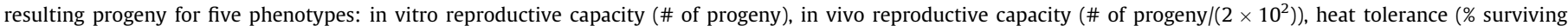

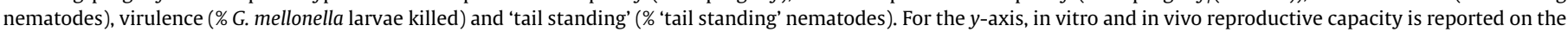

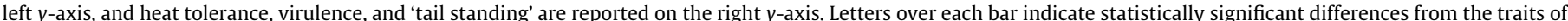

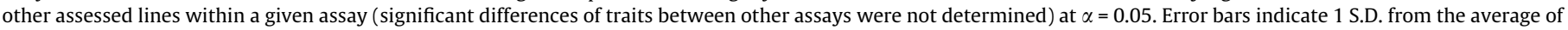
the assayed line for a given trait. 
0.0001, Fisher's LSD) than sib-cross 4 but did not differ in virulence from sib-cross 5 , and crossbred line B was similar in virulence to both sib-crosses 4 and 5 . There were no differences in 'tail standing' among any of the tested lines $(F=2.17 ; \mathrm{df}=4,34 ; P=$ 0.0.0929, Fisher's LSD). The crossbred lines had phenotypes similar to sib-cross WT in all tests except for in vitro reproductive capacity, where crossbred line B had lower in vitro reproductive capacity than sib-cross WT. Also, sib-cross WT had higher trait values than either sib-cross except for virulence and 'tail standing', whereas sib-crosses 4 and 5 were not different from sib-cross WT.

\section{Discussion}

We have shown that outcrossing laboratory-reared, trait-deteriorated lines increases trait values in progeny above the levels observed in the trait-deteriorated parental lines. Trait values in outcross and crossbred lines were both intermediate between the trait-deteriorated and WT levels (in vitro reproductive capacity), and at WT levels ('tail standing', heat tolerance, in vivo reproductive capacity). This suggests that trait deterioration in laboratoryreared $S$. carpocapsae has genetic causes. Furthermore, crossbreeding two trait-deteriorated lines recovered trait values above the levels of the corresponding sib-crosses for three tested traits (in vitro and in vivo reproductive capacity and heat tolerance), suggesting that the genetic mechanism governing trait deterioration is inbreeding rather than inadvertent laboratory selection for traits that cause poor laboratory performance. This suggests that outcrossing laboratory grown nematodes could improve production programs for EPNs in biocontrol.

Four categories of outcross trait recovery were observed in our assays: no trait deterioration to address; trait deterioration was completely recovered; trait deterioration was partially recovered; trait deterioration was not recovered. Of the 25 assays performed (five traits per five outcrosses tested) there was no trait deterioration to address in 11 assays since sib-cross WT and other sib-cross lines were similar to each other. This was primarily for virulence and 'tail standing' assays. For the remaining 14 assays, where there were differences between sib-cross WT and other sib-cross lines, outcross line traits were recovered to the level of sib-cross WT seven times (primarily for heat tolerance) or were intermediate between sib-cross WT and the relevant sib-cross line four times (primarily in vitro reproductive capacity). Traits were not recovered above the level of the relevant sib-cross line three times. Two outcrosses did not explicitly fall into any of these categories: outcross 1 virulence and outcross 5 'tail standing'. Firstly, outcross 1 virulence was lower than sib-cross 1 virulence and similar to sibcross WT virulence. Lower virulence in outcross 1 than sib-cross 1 could reflect outbreeding depression, except that outcross 1 and sib-cross WT virulence were similar. Since sib-cross 1 and sib-cross WT are similar, this result was grouped where there was no deterioration to address. Secondly, outcross 5 'tail standing' was similar to both sib-cross 5 and sib-cross WT, the only instance where an outcross was similar to the sib-cross WT and relevant sib-cross line when the sib-cross and sib-cross WT lines had significantly different values. We concluded that outcross 5 'tail standing' displayed intermediate fitness between sib-cross 3 and sib-cross WT. Overall, trait restoration above deteriorated levels in 11 of 14 tests evidences the success of outcrossing for improving traits that deteriorate during laboratory culture.

Our results indicate that inbreeding depression affected in vitro and in vivo reproductive capacity, heat tolerance and to a lesser extent 'tail standing'. Virulence and 'tail standing' traits may show little deterioration in the experimental lines because of maintenance due to laboratory selection, as these traits are under different selective pressures, or because these traits respond to laboratory growth differently than traits that were lost. Laboratory selection is likely to play a role since nematodes were propagated in insect hosts that they parasitize, maintaining some selection on virulence traits. In commercial mass production such selective pressures are unlikely to exist. 'Tail standing' is thought to be an adaptive foraging strategy (Lewis et al., 2006) and host-finding and -mortality decline when $S$. carpocapsae are unable to body wave or stand on their tails (Campbell and Gaugler, 1993), suggesting that 'tail standing' may also have been maintained by selection. Under our rearing conditions, host-finding within a $60 \mathrm{~mm}$ dish seems artificially assured, but this may not be the case. Future studies could use in vitro culture of nematode lines to assess virulence and 'tail standing' trait maintenance in the absence of selection. Alternatively, it is possible that virulence and 'tail standing' traits decreased, but our assays were not sufficiently sensitive to detect these decreases.

Currently, two models describe how inbreeding results in trait deterioration: dominance and overdominance. Each model also provides different explanations for heterosis and how to maximize trait values in commercially distributed organisms (for a recent review see Charlesworth and Willis, 2009). Under dominance, lowfrequency deleterious alleles in a population cause lower trait values in offspring and inbreeding parental lines can purge the lines of deleterious alleles. Under overdominance, heterozygotes display higher trait values than either homozygotic parent due to synergism between different alleles and commercial programs should favor heterozygote distribution. Our work confirms inbreeding as the cause of trait deterioration in S. carpocapsae. Future studies that distinguish which model of inbreeding depression is responsible for trait deterioration could inform production programs for how to best prepare $S$. carpocapsae and other EPNs for distribution. Dominance is the currently favored model, which suggests that preparation of a line purged for all deleterious alleles is a likely solution to preventing trait deterioration during batch culture. However, this outcome is not assured until it has been shown that inbreeding depression in EPNs fits the predictions of the dominance model.

Crossbreeding can distinguish the dominance and overdominance models. Under dominance, crossbreeding inbred lines should lead to trait recovery above the levels of WT lines, while overdominance should produce trait values as much as equal to, but not above, WT levels (Roff, 2002). Our study included crossbreeding experiments but lacked additional data necessary to distinguish the two models. Under dominance, crossbred line trait recovery above the level of sib-cross WT would depend upon genetic homogeneity within the experimental lines and total purging of deleterious recessive alleles from the experimental lines prior to crossing (Charlesworth and Willis, 2009). Our study lacks high-resolution genotypic data required to show genetic homogeneity of the experimental lines and purging of deleterious alleles is a slow process (Charlesworth and Willis, 2009), such that the $\sim 50-80$ generations of inbreeding in our study may not have been sufficient to purge deleterious recessive alleles. Our crossbreeding experiments showed trait values in the crossbred lines that were similar to sib-cross WT in five of the six tests where there was deterioration to address (in vivo and in vitro reproductive capacity and heat tolerance), but this finding could be observed if the lines had not been purged or were not genetically homogenous. We point out that crossbreeding experiments are generally performed only on F1 progeny, and that the only test on the F1 progeny in our study was for in vitro reproductive capacity. However, since a longterm goal for producers is to create a line that is permanently resistant to inbreeding depression we do not find it misleading to also report in vivo reproductive capacity and heat tolerance, which were tested on F12 and F3 progeny, respectively. Indeed, tests on progeny other than the F1 progeny may obfuscate trait recovery 
of virulence and nictation (tested on F9 progeny) in the F1 that was diluted by subsequent mixture among non-homogenous lines. Future studies can use the methods reported here, combined with high-resolution genotypic data, to properly test fully inbred lines of $S$. carpocapsae for trait recovery by outbreeding and crossbreeding.

One additional point should inform future studies on mechanisms of trait deterioration in EPNs. Bacteria contribute significantly to all of the phenotypes assessed in our study (Bilgrami et al., 2006) and in our study we grew each outcross on bacteria from the respective experimental line parent rather than applying a single, standardized bacterial culture to all lines. This controlled for bacterial effects when comparing the outcrosses with their respective sib-crossed counterparts (which were grown on the same bacteria as the outcrosses). However, since bacteria can make positive contributions to each of the phenotypes assessed, comparison among the outcrosses and sib-cross WT may result in lower phenotypes in the outcrosses than would be observed if the outcrosses carried WT bacteria. If this is true, the frequency of trait recovery may be even greater than we report here (recovery in 11 of 14 fitness tests).

This study has raised many additional questions that should be addressed to increase the effectiveness of culturing $S$. carpocapsae for biocontrol. Are some traits less susceptible to inbreeding depression due to an evolutionary purging of deleterious alleles or due to selection? Does the overdominance or dominance hypothesis of inbreeding depression better describe the mechanism of trait deterioration of $S$. carpocapsae? What role do bacteria play in recovery from parasitism fitness deterioration? Future studies into these and other questions are likely to increase the efficacy of commercial approaches for producing large quantities of EPNs well-suited for application as biocontrol agents.

\section{Acknowledgements}

This study was supported in part by the National Research Initiative of the United States Department of Agriculture Cooperative State Research, Education and Extension Service, Grant No. 200201974 to DIS-I, RG and BJA, and a Brigham Young University (USA) Mentored Environment Grant to BJA. JMC was supported by a National Institutes of Health (USA) National Research Service Award AI55397 in "Microbes in Health and Disease", and a National Science Foundation Graduate Research Fellowship. ARD and JMC were supported by a Brigham Young University Office of Creative Research and Activities Mentoring Grant. We thank members of the Nematode Evolution Laboratory at Brigham Young University for helpful discussion, Kathy Halat for technical assistance, and Robert Zimmerman for encouragement and inspiration.

\section{References}

Adams, B.J., Fodor, A., Koppenhofer, H.S., Stackebrandt, E., Stock, S.P., Klein, M.G., 2006. Biodiversity and systematics of nematode-bacterium entomopathogens. Biol. Control 37, 32-49.

Bai, C., Shapiro-Ilan, D.I., Gaugler, R., Hopper, K.R., 2005. Stabilization of beneficial traits in Heterorhabditis bacteriophora through creation of inbred lines. Biol. Control 32, 220-227.
Bilgrami, A.L., Gaugler, R., Shapiro-Ilan, D.I., Adams, B.J., 2006. Source of trait deterioration in entomopathogenic nematodes Heterorhabditis bacteriophora and Steinernema carpocapsae during in vivo culture. Nematology 8, 397-409.

Bird, A.F., Akhurst, R.J., 1983. The nature of the intestinal vesicle in nematodes of the family Steinernematidae. Int. J. Parasitol. 13, 599-606.

Blaxter, M.L., De Ley, P., Garey, J.R., Liu, L.X., Scheldeman, P., Vierstraete, A., Vanfleteren, J.R., Mackey, L.Y., Dorris, M., Frisse, L.M., Vida, J.T., Thomas, W.K., 1998. A molecular evolutionary framework for the phylum Nematoda. Nature 392, 71-75.

Campbell, J.F., Gaugler, R., 1993. Nictation behavior and its ecological implications in the host search strategies of entomopathogenic nematodes (Heterorhabditidae and Steinernematidae). Behaviour 126, 155-169.

Charlesworth, D., Willis, J.H., 2009. Fundamental concepts in genetics the genetics of inbreeding depression. Nat. Rev. Genet. 10, 783-796.

Dolgin, E.S., Charlesworth, B., Baird, S.E., Cutter, A.D., 2007. Inbreeding and outbreeding depression in Caenorhabditis nematodes. Evolution 61, 1339-1352.

Gaugler, R., Kaya, H.K. (Eds.), 1990. Entomopathogenic Nematodes in Biological Control. CRC Press, Boca Raton.

Gaugler, R., Campbell, J.F., 1991. Selection for enhanced host-finding of scarab larvae (Coleoptera: Scarabaeidae) in an entomopathogenic nematode. Environ. Entomol. 20, 700-706.

Grewal, P., 2002. Formulation and Application Technology. In: Gaugler, R. (Ed.), Entomopathogenic Nematodes. CABI Publishing, Wallingford, UK, pp. 265-287.

Grewal, P.S., Ehlers, R.U., Shapiro-Ilan, D.I., 2005. Nematodes as Biocontrol Agents. CABI Publishing, Oxon, UK.

Hominick, W.M., 2002. Biogeography. In: Gaugler, R. (Ed.), Entomopathogenic Nematodes. CABI Publishing, Wallingford, UK, pp. 115-143.

Hopper, K.R., Roush, R.T., Powell, W., 1993. Management of genetics of biologicalcontrol introductions. Annu. Rev. Entomol. 38, 27-51.

Kaya, H.K., Aguillera, M.M., Alumai, A., Choo, H.Y., de la Torre, M., Fodor, A., Ganguly, S., Hazir, S., Lakatos, T., Pye, A., Wilson, M., Yamanaka, S., Yang, H.W., Ehlers, R.U., 2006. Status of entomopathogenic nematodes and their symbiotic bacteria from selected countries or regions of the world. Biol. Control 38, 134-155.

Kruitbos, L.M., Wilson, M.J., 2010. Is it time to 'wave' goodbye to 'nictating' nematodes? Nematology 12, 309-310.

Lewis, E.E., Campbell, J., Griffin, C., Kaya, H., Peters, A., 2006. Behavioral ecology of entomopathogenic nematodes. Biol. Control 38, 66-79.

Martens, E.C., Heungens, K., Goodrich-Blair, H., 2003. Early colonization events in the mutualistic association between Steinernema carpocapsae nematodes and Xenorhabdus nematophila bacteria. J. Bacteriol. 185, 3147-3154.

Poinar, G.O., 1990. Taxonomy and Biology of Steinernematidae and Heterorhabditidae. In: Gaugler, R., Kaya, H.K. (Eds.), Entomopathogenic Nematodes in Biological Control. CRC Press, Boca Raton, FL, USA, pp. 23-61.

Popiel, I., Grove, D.L., Friedman, M.J., 1989. Infective juvenile formation in the insect parasite Steinernema feltiae. Parasitology 99, 77-81.

Roff, D.A., 2002. Inbreeding depression: tests of the overdominance and partial dominance hypotheses. Evolution 56, 768-775.

Schmeige, D.C., 1963. The feasibility of using a neoaplectanid nematode for control of some forest insect pests. J. Econ. Entomol. 5, 427-431.

Shapiro, D.I., Glazer, I., Segal, D., 1996. Trait stability and fitness of the heat tolerant entomopathogenic nematode Heterorhabditis bacteriophora IS5 strain. Biol. Control 8, 153-159.

Shapiro-Ilan, D.I., Gaugler, R., 2002. Production technology for entomopathogenic nematodes and their bacterial symbionts. J. Ind. Microbiol. Biotechnol. 28, 137146.

Shapiro-Ilan, D.I., Gouge, D.H., Koppenhofer, A.M., 2002. Factors Affecting Commercial Success: Case Studies in Cotton, Turf and Citrus. In: Gaugler, R. (Ed.), Entomopathogenic Nematology. CABI, New York, NY, pp. 333-356.

Snyder, H., Stock, S.P., Kim, S.K., Flores-Lara, Y., Forst, S., 2007. New insights into the colonization and release processes of Xenorhabdus nematophila and the morphology and ultrastructure of the bacterial receptacle of its nematode host, Steinernema carpocapsae. Appl. Environ. Microbiol. 73, 5338-5346.

Southwood, T.R.E., 1978. Ecological Methods. Chapman and Hall, London.

Stuart, R.J., Gaugler, R., 1996. Genetic adaptation and founder effect in laboratory populations of the entomopathogenic nematode Steinernema glaseri. Can. J. Zool. 74, 164-170

Vivas, E.I., Goodrich-Blair, H., 2001. Xenorhabdus nematophilus as a model for hostbacterium interactions: $r p o S$ is necessary for mutualism with nematodes. J. Bacteriol. 183, 4687-4693.

Wang, J.X., Bedding, R.A., 1996. Population development of Heterorhabditis bacteriophora and Steinernema carpocapsae in the larvae of Galleria mellonella. Fund. Appl. Nematol. 19, 363-367.

White, G.F., 1927. A method for obtaining infective nematode larvae from cultures. Science 66, 302-303. 